INPLASY

PROTOCOL

To cite: Cai et al. Meta-analysis of blood lipid reduction for patients with coronary heart disease by combination of pitavastatin and ezetimibe. Inplasy protocol 202150072. doi:

10.37766/inplasy2021.5.0072

Received: 18 May 2021

Published: 18 May 2021

Corresponding author: Qiang Su

drsuqiang@163.com

Author Affiliation: Department of Cardiology, Affiliated Hospital of Guilin Medical University, Guilin, China.

Support: None.

Review Stage at time of this submission: Data analysis.

Conflicts of interest:

None declared.

\section{Meta-analysis of blood lipid reduction for patients with coronary heart disease by combination of pitavastatin and ezetimibe}

Cai, R' Xu, Y2; Su, Q³.

Review question / Objective: How does pitavastatin combined with ezetimibe effect the blood lipids in patients with coronary heart disease.

Condition being studied: Coronary heart disease (CHD) is a cardiovascular disease and the main cause of death in developed and developing countries. According to a report submitted by the World Health Organization in 2009, 17.3 million people die from cardiovascular disease every year. CHD caused a 6 million reduction in the population of the United States in 2005. In the past 10 years, the hospitalization rate of coronary heart disease in China has increased by three times, and its morbidity and mortality will continue to increase in the next 10 years. The huge burden caused by cardiovascular disease has become a very important public health problem. Risk factors for coronary heart disease include gender, age, dyslipidemia, smoking, diabetes, high blood pressure, obesity, etc. The most important of which is dyslipidemia. Studies have shown that elevated low-density lipoprotein cholesterol (LDL-C) is one of the independent risk factors for coronary heart disease. Statins are the cornerstone of lowering LDL-C. Regardless of coronary heart disease, statins can reduce cardiovascular events by affecting LDL-C . Every $1 \mathrm{mmol} / \mathrm{L}$ decrease in LDL-C reduces coronary events by $24 \%$, stroke events by $15 \%$, and coronary artery reconstruction events by $24 \%$.

INPLASY registration number: This protocol was registered with the International Platform of Registered Systematic Review and Meta-Analysis Protocols (INPLASY) on 18 May 2021 and was last updated on 27 May 2021 (registration number INPLASY202150072).

\section{INTRODUCTION}

Review question / Objective: How does pitavastatin combined with ezetimibe effect the blood lipids in patients with coronary heart disease. 
Condition being studied: Coronary heart disease (CHD) is a cardiovascular disease and the main cause of death in developed and developing countries. According to a report submitted by the World Health Organization in 2009, 17.3 million people die from cardiovascular disease every year. CHD caused a 6 million reduction in the population of the United States in 2005. In the past 10 years, the hospitalization rate of coronary heart disease in China has increased by three times, and its morbidity and mortality will continue to increase in the next 10 years. The huge burden caused by cardiovascular disease has become a very important public health problem. Risk factors for coronary heart disease include gender, age, dyslipidemia, smoking, diabetes, high blood pressure, obesity, etc. The most important of which is dyslipidemia. Studies have shown that elevated low-density lipoprotein cholesterol (LDL-C) is one of the independent risk factors for coronary heart disease. Statins are the cornerstone of lowering LDL-C. Regardless of coronary heart disease, statins can reduce cardiovascular events by affecting LDL-C . Every $1 \mathrm{mmol} / \mathrm{L}$ decrease in LDL-C reduces coronary events by $24 \%$, stroke events by $15 \%$, and coronary artery reconstruction events by $24 \%$.

\section{METHODS}

Participant or population: Inclusion: Patients with coronary heart disease. Exclusion:Patient has no coronary heart disease.

Intervention: Pitavastatin+eaetimibe +current background therapy.

Comparator: Pitavastatin+current background therapy.

Study designs to be included: We will include all randomized controlled trials (RCT).

Eligibility criteria: The inclusion criteria were as follows: (1) all randomized controlled trials; (2) follow-up time of $\geq 8$ weeks; (3) age $\geq 18$ years; (4) the test group given oral pitavastatin and ezetimibe and the control group given oral pitavastatin based on the standard treatment of CHD; and (5) diagnosis of CHD meeting the definition in the included literature.

Information sources: Electronic databases, including PubMed, Cochrane Library, Embase, NCKI, VIP, and WanFang Data, were searched from their establishment to October 8, 2020, for relevant studies using the following keywords: pitavastatin/ pitavastatin calcium/pitavastatin lactone/ (E, 3R, 5S)-7-(2-cyclopropyl-4-(4fluorophenyl) quinolin - 3-yl) - 3,5 dihydroxyhept-6-enoic acid/P-872441/ NK-104/itavastatin/itavastatin calcium/ nisvastatin, ezetimibe/(1-(4-fluorophenyl)( 3 R ) - ( 3 - ( 4 - fl u o r o p h e n y I) - ( 3 S ) hydroxypropyl)-(4S)-(4-hydroxyphenyl)-2azetidinone)/ezetrol/SCH-58235/zetia, coronary heart disease/CHD. Chinese and English literature, as well as reference lists in other related reports, were searched to identify other potentially relevant data.

Main outcome(s): The primary endpoint is the levels of serum LDL-C, TC, TG and HDL-C in patients after treatment.

\section{Additional outcome(s): creatine kinase.}

Quality assessment / Risk of bias analysis: The consistency of the RCTs was measured using the prejudice risk evaluation method described in Cochrane Handbook 5.1.0 [15], and the following criteria have been evaluated: random sequence generation, allocation concealment, personnel, and patient blindness, insufficient outcome records, selective coverage, the blindness of outcomes evaluation, other bias. Studies were determined to have a strong degree of high, low or unclear, based on the above 7 objects.

Strategy of data synthesis: Stata16 software was used for Meta analysis. The measurement data were analyzed and statistic using standardized mean difference (SMD) and its $95 \%$ confidence interval. The SMD and $95 \% \mathrm{Cls}$ of serum lipid concentrations in the test group and control group were defined as intervention 
effects, and random effects models were used to evaluate the combined effects. The $P$ value of heterogeneity is calculated to evaluate whether there is statistical heterogeneity between studies. $P>0.1$ means that there is no statistical heterogeneity between studies. On the contrary, $\mathrm{P} 50 \%$ indicates significant heterogeneity between studies. According to the participant's follow-up time ( $\leq$ VS.>12weeks) and health status (diabeticVS.non-diabetic) for subgroup analysis. Sensitivity analysis is carried out by changing the effect model and removing the test piece by piece. Egger was used to test publication bias. $\mathrm{P}>0.05$ indicated no obvious publication bias, and $\mathrm{P}<0.05$ indicated there was publication bias. In the presence of publication bias, a Trimfill analysis is performed to detect the impact of the bias on the overall effect.

Subgroup analysis: According to the participant's follow-up time ( $\leq$ VS. $>12$ weeks) and health status (diabeticVS.non-diabetic) for subgroup analysis.

Sensitivity analysis: The random-effects model was changed to a fixed-effects model for sensitivity analysis. The results showed that the combination of pitavastatin and ezetimibe caused no significant changes in the overall effect of LDL-C (SMD $=-0.77,95 \% \mathrm{Cl}=-0.86$ to $0.68, \mathrm{P}<0.01), \mathrm{TC}$ (SMD $=-0.79,95 \% \mathrm{Cl}=-$ 0.93 to $-0.65, \mathrm{P}<0.01$ ), TG (SMD $=-0.57$, $95 \% \mathrm{Cl}=-0.72$ to $-0.42, \mathrm{P}<0.01$ ), and HDLC $(\mathrm{SMD}=0.39,95 \% \mathrm{Cl}=0.25-0.53, \mathrm{P}<$ 0.001 ) levels, suggesting that the original meta-analysis results had a certain degree of stability and were highly reliable. An individual study was eliminated to determine whether it changed the combined effect. After eliminating each study, the overall effect of the test group on LDL-C, TC, TG, and HDL-C levels did not change significantly. Egger's test found no publication bias in the LDL-C (Egger's: $P=$ 0.196), TG (Egger's: $P=0.487$ ), and HDL-C levels (Egger's: $P=0.06$ ), but had an obvious publication bias in the TC level (Egger's: $\mathrm{P}<0.0001$ ). According to the trimand-fill analysis, a study needed to be added to the right side of the TC study to balance the asymmetry. After adjustment by the trim-and-fill analysis, the TC level (SMD $=-0.780,95 \% \mathrm{Cl}=-1.042$ to -0.518 ) still showed a significant decrease.

Country(ies) involved: China.

Keywords: Pitavastatin; Ezetimibe; Coronary heart disease; Blood lipid.

\section{Contributions of each author:}

Author 1 - Ruping Cai - The author has made substantial contributions to conception and design of the study; Author 1 and Author 2 searched literature, extracted data from the collected literature and analyzed the data; Author 1 wrote the manuscript; All authors approved the final version of the manuscript.

Email: crp314689269@163.com

Author 2 - Yuli Xu - Author 1 and Author 2 searched literature, extracted data from the collected literature and analyzed the data; All authors approved the final version of the manuscript.

Email: 869553991@qq.com

Author 3 - Qiang Su - The author revised the manuscript; All authors approved the final version of the manuscript.

Email: drsuqiang@163.com 